\title{
Brentuximab vedotin for relapsed or refractory Hodgkin lymphoma: experience in Turkey
}

\author{
A. Salihoglu • T. Elverdi • I. Karadogan • S. Paydas • E. Ozdemir • G. Erdem • \\ N. Karadurmus • G. Akyol • L. Kaynar • ZA Yegin • G. Sucak • V. Ozkocaman • P. Topcuoglu • \\ M. Ozcan • E. Birtas • H. Goker • Z. Baslar • B. Ferhanoglu
}

Received: 26 January 2014 / Accepted: 6 September 2014 /Published online: 18 September 2014

(C) The Author(s) 2014. This article is published with open access at Springerlink.com

\begin{abstract}
Current treatment modalities can cure up to 70 $80 \%$ of patients with classical Hodgkin lymphoma. Approximately, $20-30 \%$ of patients require further treatment options. Brentuximab vedotin has been approved for the treatment of relapsed and refractory Hodgkin lymphoma. In the present study, we report the experience with brentuximab vedotin as single agent in 58 patients with relapsed or refractory Hodgkin lymphoma. The objective response rate was $63.5 \%$ with 13 complete responders (26.5\%) among 49 patients evaluated at the early phase of treatment ( $2-5$ cycles). Upon treatment prolongation ( $\geq 6$ cycles), 37 patients achieved a final objective response rate of $32.4 \%$ with $21.6 \%$ of complete and $10.8 \%$ of partial response. Overall survival at 12 months was
\end{abstract}

\section{A. Salihoglu $\cdot$ T. Elverdi $\cdot$ Z. Baslar}

Cerrahpasa Medical Faculty, Department of Internal Medicine,

Division of Hematology, Istanbul University, Istanbul, Turkey

I. Karadogan

Division of Hematology, Antalya Medstar Hospital, Antalya, Turkey

\section{S. Paydas}

Department of Internal Medicine, Division of Medical Oncology,

Cukurova University, Adana, Turkey

\section{E. Ozdemir}

Institute of Oncology, Stem Cell Transplantation Unit,

Hacettepe University, Ankara, Turkey

\section{G. Erdem $\cdot$ N. Karadurmus}

Department of Internal Medicine, Gulhane Military Medical

Academy, Division of Medical Oncology, Ankara, Turkey

\section{G. Akyol $\cdot$ L. Kaynar}

Erciyes University, Department of Internal Medicine,

Division of Hematology, Kayseri, Turkey

\section{Z. Yegin · G. Sucak}

Department of Internal Medicine, Division of Hematology,

Gazi University, Ankara, Turkey
$70.6 \%$, and progression-free survival at 12 months was $32.8 \%$. Median overall survival could not be reached and median progression-free survival was 7 months. While the median duration of response was 9 months in the whole cohort, it was 11.5 months in the complete responders. Complete response rates in patients treated with $>3$ chemotherapy regimens before brentuximab vedotin were significantly lower $(p=0.016)$. Fourteen patients were subsequently transplanted. In conclusion, brentuximab vedotin provided a bridge to transplantation in approximately one quarter of the patients. The declining response rates during the course of treatment suggest that transplantation should be implemented early during brentuximab vedotin treatment.

\section{Ozkocaman}

Department of Internal Medicine, Division of Hematology,

Uludag University, Bursa, Turkey

P. Topcuoglu $\cdot$ M. Ozcan

Department of Internal Medicine, Division of Hematology,

Ankara University, Ankara, Turkey

E. Birtas

Kocaeli University, Department of Internal Medicine,

Division of Hematology, Kocaeli, Turkey

H. Goker

Hacettepe University, Department of Internal Medicine, Division of Hematology, Ankara, Turkey

B. Ferhanoglu $(\bowtie)$

Koc University, Department of Internal Medicine,

Division of Hematology, Istanbul, Turkey

e-mail: bferhan@superonline.com

B. Ferhanoglu

e-mail: bferhan@gmail.com 
Keywords Brentuximab vedotin · Hodgkin Lymphoma . Relapsed $\cdot$ Refractory

\section{Introduction}

Classical Hodgkin lymphoma (cHL) is one of the most curable cancers. However, approximately a third of patients will relapse after first-line therapy and $15 \%$ will fail both first and second line therapies, and therapeutic options are still quite limited in these patients [1]. Brentuximab vedotin (BV) is a novel antibody-drug conjugate composed of a CD30-directed recombinant chimeric immunoglobulin G1 covalently linked to a synthetic antitubulin chemotherapeutic agent monomethyl auristatin E. BV showed an impressive activity against relapsed/refractory cHL [2].

We would like to provide more information on the efficacy and safety of $\mathrm{BV}$ and contribute to the determination of the best role and timing of BV treatment in the current study.

\section{Design and methods}

$\mathrm{BV}$ is approved for the treatment of $\mathrm{cHL}$ after failure of autologous transplantation or after failure of at least two prior multi-agent chemotherapy regimens in patients that are not candidates for transplantation. Eleven Turkish institutions participated in this multicenter, retrospective study. Eligible patients were required to be treated with at least two courses of $\mathrm{BV}$ and to have available clinical documentation and staging investigations. Inclusion of the patients with organ dysfunction was based on the decision of the attending physician.

All patients underwent baseline assessments including physical examination, routine laboratory tests, and radiological examinations [computed tomography (CT), positron emission tomography (PET/CT)] prior to the treatment. Patients were included without any limitations regarding performance status and organ function. All of the patients provided written informed consent.

$\mathrm{BV}$ is imported via the named-patient-program. BV was given at a dose of $1.8 \mathrm{mg} / \mathrm{kg}$ intravenous infusion over $30 \mathrm{~min}$ every 3 weeks in an outpatient setting. Since the drug is received institutionally, the relative dose intensity was calculated as total chemotherapy dose delivered/total chemotherapy duration [3].

The primary endpoint of the study was the objective response rate (ORR); secondary endpoints were safety, overall survival (OS), and progression-free survival (PFS). Response was assessed by PET/CT or CT early during disease course after $2-5$ cycles [ 4$]$ and after $\geq 6$ cycles using the international working group revised response criteria [5].

Safety and tolerability were assessed before each BV cycle according to National Cancer Institute Common Terminology
Criteria for Adverse Events Version 4.0. For grade 3 toxicity, dose reduction of $\mathrm{BV}$ to $1.2 \mathrm{mg} / \mathrm{kg}$ was recommended.

OS was defined as the time from the initiation of $\mathrm{BV}$ to death of any cause. PFS was the time from treatment initiation to progression, relapse, or death of any cause [5]. Primary refractory disease was defined as no complete remission or relapse within 3 months of first line therapy [6]. Both OS and PFS were censored at the date of last information. Median duration of response, PFS, and OS rates along with two-sided $95 \%$ confidence interval (CI) was estimated using the Kaplan-Meier method. Survival functions were compared using log-rank test. Analysis of the data was carried out using STATA 11.1 SE software.

\section{Results}

From March 2011 to July 2013, 58 patients in eleven Turkish centers were treated with BV within the named patient program. Outcomes and safety/toxicity data of 58 patients with relapsed or refractory $\mathrm{cHL}$ were analyzed in this study, aiming to evaluate the efficacy and safety of BV.

Demographics and disease characteristics of the patients are summarized in Table 1. The median age at diagnosis was 26 years, and $64 \%$ were males. Nodular sclerosis was the most frequent (79\%) histologic subtype, half of the patients (49\%) had primary refractory disease, and $72 \%$ were refractory to the last treatment given before BV. Most of the patients $(77.5 \%)$ had advanced stage disease and approximately half of them (47\%) had B-symptoms.

A median of 7 (range, 2-18) courses of BV were given as a single agent (Fig. 1) and relative dose intensity was calculated as $81.6 \%$. The results of post-BV assessments are shown in Fig. 2. PET/CT was performed in 36 patients and 13 were assessed with CT early during treatment course. Thirty-one patients underwent PET/CT and 6 were evaluated with $\mathrm{CT}$ scan after $\geq 6$ cycles of BV.

Early assessment after $2-5$ cycles of BV

After 2-5 cycles of BV, an objective response rate (ORR) of $63.5 \%(n=31)$ with $26.5 \%(n=13) \mathrm{CR}$ was achieved.

In the subgroup of patients with an objective response, the median PFS duration was 9 months and PFS at 12 months was $44.34 \%$ (95\% CI, 24.6 to $62.5 \%$ ). In the remaining 18 patients without an objective response, PFS was significantly lower $(p<0.001)$ with a median PFS of 3 months. OS at 12 months was $80.5 \%$ (95\% CI, 59.0 to $91.5 \%$ ) and $57.9 \%$ (95\% CI, 28.6 to $78.8 \%$ ) in patients with or without objective response, respectively. The median OS has not been reached in either group.

Among the 13 patients who achieved CR at the early evaluation after 2-5 treatment cycles, the frontline treatment 
Table 1 Patient demographics and disease characteristics $(N=58)$

\begin{tabular}{|c|c|c|c|}
\hline \multicolumn{3}{|l|}{ Median age at diagnosis, years (range) } & $26(13-62)$ \\
\hline \multirow{2}{*}{\multicolumn{3}{|c|}{ Sex, male/female, $n / n$}} & $37 / 21$ \\
\hline & & & $n(\%)$ \\
\hline \multicolumn{3}{|l|}{ Systemic symptoms at diagnosis ${ }^{\mathrm{a}}$} & $46(82)$ \\
\hline \multirow[t]{4}{*}{ Histological subtype } & \multicolumn{2}{|l|}{ Nodular sclerosis } & $46(79)$ \\
\hline & \multicolumn{2}{|l|}{ Mixed cellularity } & $6(10)$ \\
\hline & \multicolumn{2}{|l|}{ Lymphocyte rich } & $1(2)$ \\
\hline & \multicolumn{2}{|l|}{ Not determined } & $5(9)$ \\
\hline \multicolumn{3}{|l|}{ Frontline Hodgkin lymphoma regimen ABVD } & $57(98)$ \\
\hline \multicolumn{3}{|l|}{ Prior radiation therapy } & $45(77.5)$ \\
\hline \multirow[t]{4}{*}{ Prior transplantation $(N=49)^{\mathrm{b}}$} & \multicolumn{2}{|l|}{ autologous } & $39(79.6)$ \\
\hline & \multicolumn{2}{|l|}{ allogeneic } & $1(2.05)$ \\
\hline & \multicolumn{2}{|c|}{ autologous + allogeneic } & $8(16.3)$ \\
\hline & \multicolumn{2}{|c|}{ autologous + haploidentical allogeneic } & $1(2.05)$ \\
\hline Median prior chemotherapy regimens & & & $4(2-7)$ \\
\hline Primary refractory disease to frontline therapy & & & $28(49)$ \\
\hline Refractoriness to most recent therapy & & & $42 / 58(72)$ \\
\hline \multirow[t]{5}{*}{ Disease status at the initiation of brentuximab vedotin } & \multicolumn{2}{|l|}{ Stage III/IV } & $45(77.5)$ \\
\hline & \multicolumn{2}{|l|}{ B-symptoms } & $27(47)$ \\
\hline & Performance status & ECOG score 0 & $14(24)$ \\
\hline & & ECOG score 1 & $32(55)$ \\
\hline & & ECOG score 2 & $12(21)$ \\
\hline
\end{tabular}

$A B V D$ adriamycin + bleomycin + vinblastine + dacarbazine, ECOG Eastern Cooperative Oncology Group

${ }^{a}$ Two patients did not have systemic symptom evaluation results

${ }^{\mathrm{b}}$ Nine patients $(15 \%)$ had not undergone stem cell transplantation previously because of progressive disease (PD) in seven, poor mobilization in one, and decision of the attending physician in another regimen was ABVD (adriamycin + bleomycin + vinblastine + dacarbazine) and the median number of BV courses was nine. Other characteristics of these patients are given in Table 2. Of these 13 patients, one died due to infection; five underwent transplantation (three allogeneic, one haploidentical, and one autologous) after BV with a follow-up time of 1 month for two patients; 7, 8, and 12 months for three patients after transplantation; four could not find a matched donor and proceed to transplantation; one refused transplantation although there was a matched sibling donor; and one was preparing for allogeneic transplantation at the time of statistical analysis; one patient was not a transplant candidate, because allogeneic transplantation had been already performed.
Five patients were referred to transplantation before 6 cycles of therapy. Three of them underwent allogeneic and one of them autologous stem cell transplantation. The fifth patient had not been transplanted yet at the time of statistical analysis.

Twenty-one patients did not undergo a later assessment of response after $\geq 6$ cycles of treatment. Of these, 11 had disease progression during early treatment course and discontinued therapy, one died because of infection and two were awaiting response evaluation at the time of statistical analysis. Response assessment was omitted in two patients after achieving complete response (CR) during early response evaluation since these patients did not have any detectable clinical evidence of disease and disease-related symptoms. They were
Fig 1 Number of brentuximab vedotin course

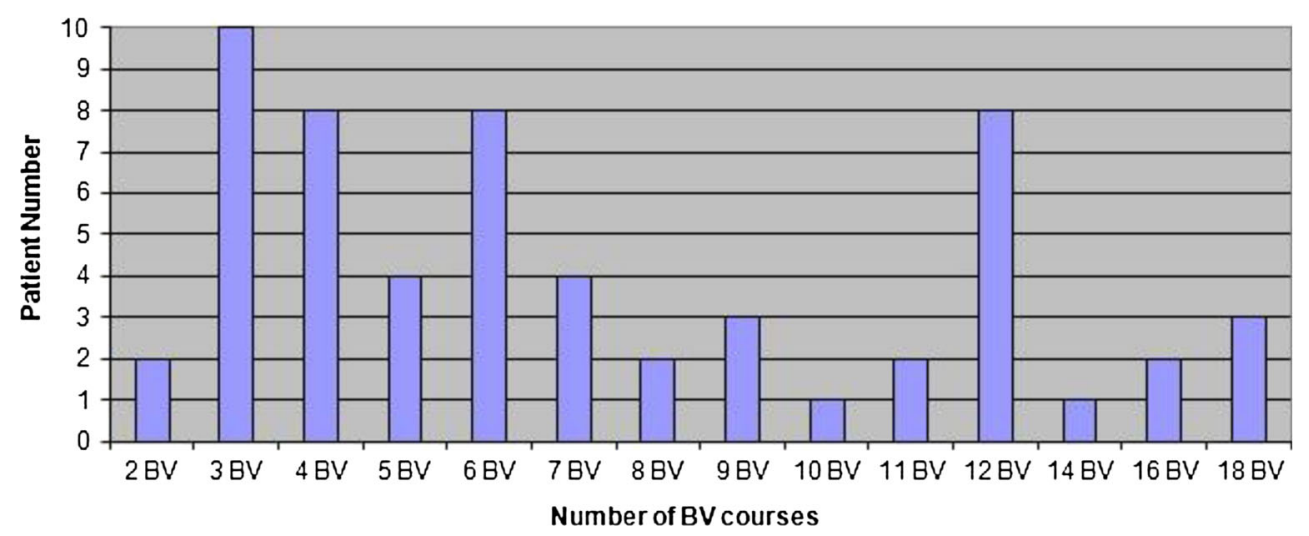


Fig 2 Results of post-BV assessments

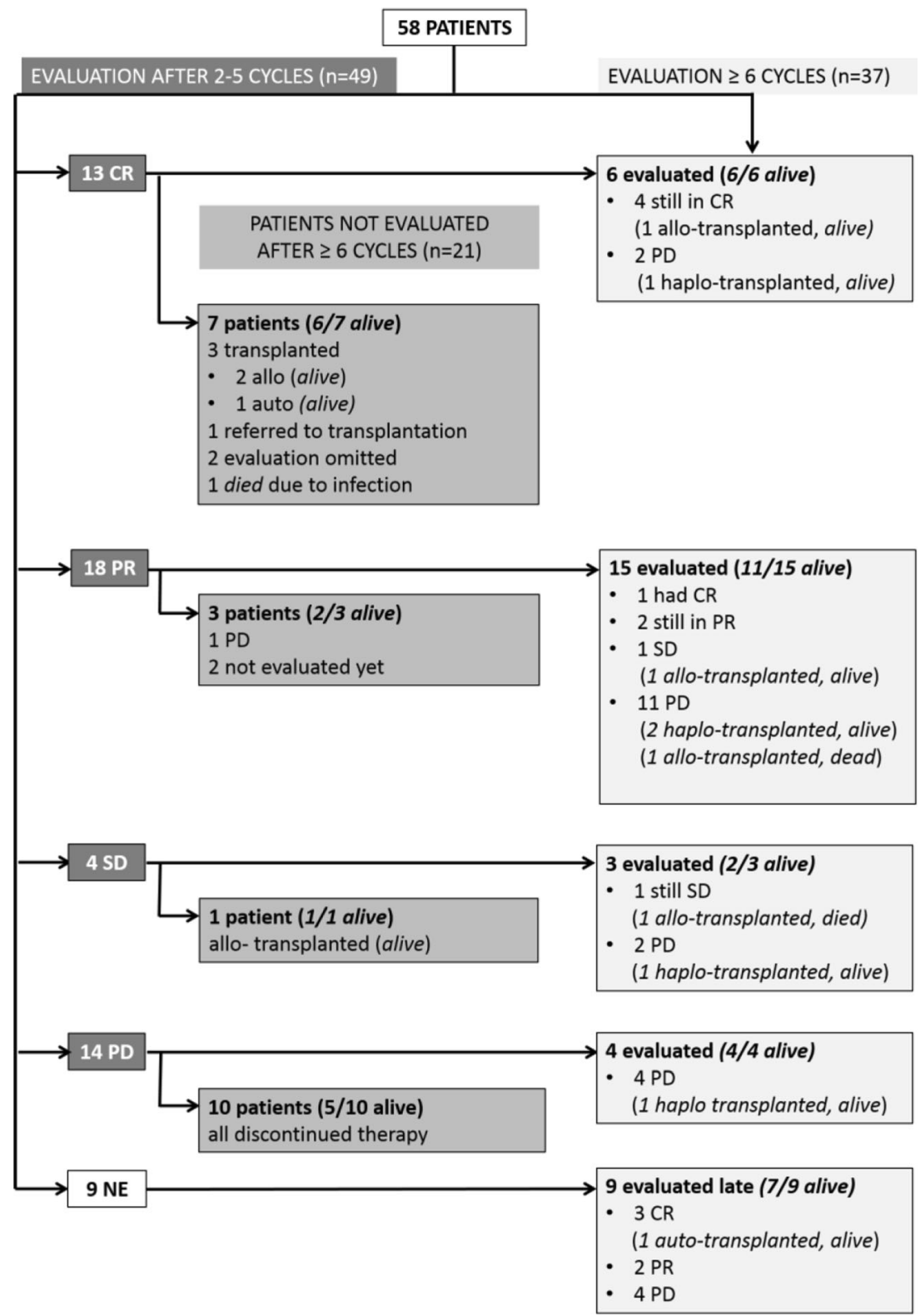

treated with 18 courses of BV but could not proceed with transplantation (one patient had no donor and the other had autologous and allogeneic transplantation before BV).

Table 2 Characteristics of 13 patients who achieved complete remission at early evaluation, after $2-5$ cycles of brentuximab vedotin

\begin{tabular}{ll}
\hline Median age at diagnosis, years (range) & $25(16-53)$ \\
Sex, male/female, $n / n$ & $9 / 4$ \\
$\begin{array}{l}\text { Prior radiation therapy, } n(\%) \\
\text { Prior transplantation, } n(\%)\end{array} \quad 11(85)$ \\
$\quad \quad$ autologous stem cell & $9(69)$ \\
Number of previous chemotherapy regimens, median (range) & $4(2-6)$ \\
Refractory to frontline therapy, $n(\%)$ & $3(23)$ \\
Refractory to most recent therapy, $n(\%)$ & $8(61.5)$ \\
\hline
\end{tabular}

Later assessment (after $\geq 6$ cycles of BV)

Nine patients were not evaluated (NE) early during treatment course based on the attending physician's decisions (three achieved CR, two achieved partial remission (PR), and four were found to have PD), but evaluated after $\geq 6$ cycles.

Response assessment after $\geq 6$ cycles was performed with $\mathrm{PET} / \mathrm{CT}$ in 31 of 37 patients and CT in 6 patients and showed an ORR of $32.4 \%(n=12)$ with CR in 8 patients (21.6\%). PFS at 12 months was $32.8 \%$ (95\% CI, 19.7 to $46.6 \%)$ and the median PFS duration was 7 months (95\% CI, 4.8 to $11.3 \%$ ) (Fig. 3a). Fourty-four patients were alive at the time of data analysis. OS at 12 months was $70.6 \%$ (95\% CI, 54.4 to $81.9 \%$ ) and the median OS has not been reached yet (Fig. 3b). 

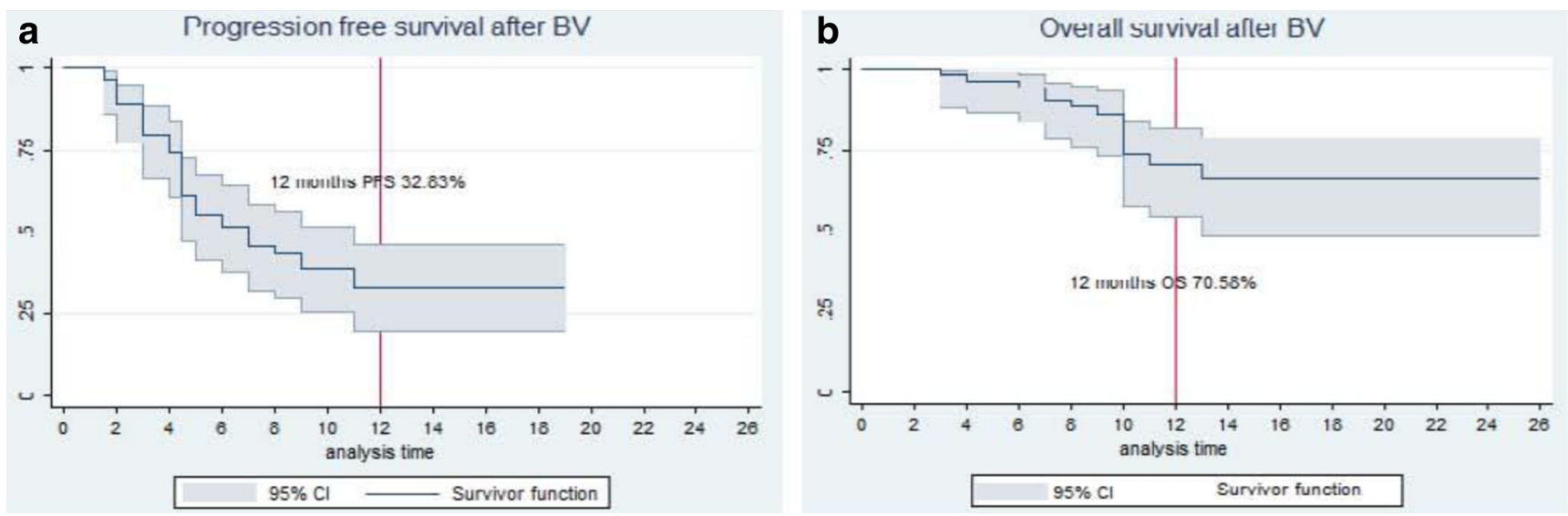

Fig 3 Kaplan-Meier plots and $95 \%$ CIs for PFS and OS. a The median PFS was 7 months. b The OS at 12 months was $70.58 \%$. The median OS has not yet been reached

Twelve patients had been treated with three or less previous chemotherapy regimens before $\mathrm{BV}$ and half of the patients achieved CR. CR rates in patients treated with $>3$ chemotherapy regimens before $\mathrm{BV}$ were significantly lower $(15 \%)$ than $\mathrm{CR}$ rates in patients with fewer prior therapies $(p=0.016)$.

Fourteen patients proceeded to transplantation ( 7 allogeneic, 5 haploidentical, and 2 autologous transplantation). Among these, 5 patients ( $36 \%$ ) entered transplantation in $\mathrm{CR}$, while 3 (21\%) with stable disease (SD) and $6(43 \%)$ with PD (Table 3). After transplantation, 12 patients (85\%) survived. All five patients receiving transplantation in CR (3 allogeneic, 1 autologous, and 1 haploidentical) were in continuous CR after $1,1,7,8$, and 12 months of transplantation, respectively. None of the patients entering transplantation with SD or PD $(n=9)$ could achieve remission after transplantation.

\section{Safety/toxicity}

In general, the treatment was well tolerated with dose reduction required in five patients (three due to cytopenias, one peripheral neuropathy, one unknown). The most common $(>10 \%)$ treatment-related adverse events were fatigue, nausea, neuropathy, neutropenia, vomiting, myalgia, alopecia, and extremity pain (Table 4).

Neurological toxicity was observed in 20 patients: peripheral neuropathy pattern in 17 , grade 3-4 neuropathy occurred in two, and oculomotor nerve palsy in one patient. Two patients from different centers suffered from generalized tonic convulsions, one of whom was on renal replacement therapy. Convulsions occurred after 5 and 2 cycles of BV treatment.

Table 3 Disease status at transplantation

\begin{tabular}{ll}
\hline & $N(\%)$ \\
\hline Complete remission & $5(36)$ \\
Stable disease & $3(21)$ \\
Progressive disease & $6(43)$
\end{tabular}

Since drug-induced neurotoxicity could not be excluded, BV was stopped in both patients.

\section{Discussion}

This multicenter, retrospective study was conducted to investigate activity and safety of BV in patients with cHL. Patients were managed in a non-trial setting, and the study population mainly consisted of heavily pretreated, high-risk patients. Although having multiply relapsed or refractory disease, ECOG performance status of the patients was $\leq 2$.

In general, treatment was well tolerated, and toxicities were generally grade 1 and 2 in severity. Patients with organ dysfunction were not excluded. Namely, there were three patients with chronic kidney disease (one with stage 4 and two with stage 5 , undergoing hemodialysis) included in the study. All three patients had received previous salvage chemotherapy

Table 4 Most common adverse events reported by $>10 \%$ of the patients regardless of relationship with brentuximab vedotin

\begin{tabular}{lll}
\hline Adverse event (AE) & $\begin{array}{l}\text { Total number of patients } \\
\text { with AE, } n(\%)\end{array}$ & $\begin{array}{l}\text { Grade 3-4 AE, } \\
n(\%)\end{array}$ \\
\hline Fatigue & $29(50.0)$ & - \\
Nausea & $19(32.8)$ & $2(3.4)$ \\
Neuropathy & $18(31.0)$ & $2(3.4)$ \\
Neutropenia & $16(27.6)$ & $1(1.7)$ \\
Vomiting & $15(25.8)$ & $1(1.7)$ \\
Myalgia & $15(25.8)$ & $2(3.4)$ \\
Alopecia & $12(20.7)$ & - \\
Extremity pain & $12(20.7)$ & $2(3.4)$ \\
Pyrexia & $8(13.8)$ & - \\
Muscle spasm & $8(13.8)$ & - \\
Constipation & $7(12.0)$ & - \\
Pruritus & $7(12.0)$ & - \\
\hline
\end{tabular}


including cisplatin. Two of these patients were treated without any dose modifications. One of these hemodialysis patients treated without dose reduction had generalized seizures after two cycles of BV and therapy was stopped. The other two patients with stage 4-5 chronic kidney disease did not experience any particular side effects or deterioration of kidney function during treatment course.

Interestingly, generalized seizures with a toxicity grade of 3 were reported in two patients from two different centers. One of the patients had stage 5 chronic kidney disease and seizures occurred after two cycles as mentioned previously. The other patient experienced seizures after 5 treatment cycles and treatment was stopped. Grade 3 oculomotor nerve disorder developed in one patient after 7 BV treatment cycles. Possible underlying causes were excluded in this patient. One patient with a previous diagnosis of demyelinating polyneuropathy did not experience any deterioration of neuropathy during the treatment course.

The retrospective nature of the analysis of data was a limitation of the present study.

The finding that patients with less prior chemotherapy regimens had better CR rates compared with more heavily pretreated patients $(p=0.016)$ should be interpreted cautiously because of the low number of less intensively treated patients and no data in the literature supporting this finding.

Similar to previous studies [6-9], our study also indicates that best responses to $\mathrm{BV}$ are observed after $2-5$ cycles, early during the treatment course. Since best results are obtained with chemosensitive disease and minimal tumor burden both in autologous and allogeneic stem cell transplantation, transplantation should be considered earlier during BV treatment when best responses are achieved.

In the present study, nearly one quarter of patients $(n=14)$ underwent transplantation (7 allogeneic, 5 haploidentical and 2 autologous) after BV therapy, which is consistent with the current literature $[8,10]$. However, only $36 \%$ of these patients $(n=5)$ were in complete remission before transplantation, and $43 \%$ of patients $(n=6)$ were transplanted with chemoresistant PD. This poor pre-transplant disease control showed that BV was not used as an effective bridge to transplant in the present study. In spite of this fact, $85 \%$ of the transplanted patients were alive. Consideration of transplantation early during BV treatment course can improve disease status at transplantation.

Acknowledgment BF designed the study. AS and BF performed the research, collected the data, analyzed the data, and wrote the paper. TE, IK, SP, EO, GE, NK, GA, LK, ZAY, GS, VO, PT, MO, EB, HG, and ZB collected the data. All authors read the paper. SP and GS reviewed the paper and contributed to the content of the paper. Thanks to Murat OZBALAK, M.D. for his support during the preparation of the paper.

Conflicts of interests Burhan Ferhanoglu was a member of advisory committee for Millennium The Takeda Oncology Company and received honorarium from Millenium The Takeda Oncology.
Open Access This article is distributed under the terms of the Creative Commons Attribution License which permits any use, distribution, and reproduction in any medium, provided the original author(s) and the source are credited.

\section{References}

1. Skarbnik AP, Pro B (2013) Heads or tails? Choosing a salvage therapy for relapsed/refractory Hodgkin lymphoma. Expert Rev Hematol 6(1):1-3. doi:10.1586/ehm.12.71

2. Gualberto A (2012) Brentuximab Vedotin (SGN-35), an antibodydrug conjugate for the treatment of CD30-positive malignancies. Expert Opin Investig Drugs 21(2):205-216. doi:10.1517/13543784. 2011.641532

3. Bosly A, Bron D, Van Hoof A, De Bock R, Berneman Z, Ferrant A, Kaufman L, Dauwe M, Verhoef G (2008) Achievement of optimal average relative dose intensity and correlation with survival in diffuse large B-cell lymphoma patients treated with CHOP. Ann Hematol 87(4):277-283. doi:10.1007/s00277-007-0399-y

4. Kahraman D, Theurich S, Rothe A, Kuhnert G, Sasse S, Scheid C, Dietlein M, Drzezga A, von Bergwelt-Baildon M, Kobe C (2013) 18Fluorodeoxyglucose positron emission tomography/computed tomography for assessment of response to brentuximab vedotin treatment in relapsed and refractory Hodgkin lymphoma. Leuk Lymphoma. doi:10.3109/10428194.2013.819575

5. Cheson BD, Pfistner B, Juweid ME, Gascoyne RD, Specht L, Horning SJ, Coiffier B, Fisher RI, Hagenbeek A, Zucca E, Rosen ST, Stroobants S, Lister TA, Hoppe RT, Dreyling M, Tobinai K, Vose JM, Connors JM, Federico M, Diehl V (2007) Revised response criteria for malignant lymphoma. J Clin Oncol 25(5):579-586

6. Younes A, Gopal AK, Smith SE, Ansell SM, Rosenblatt JD, Savage KJ, Ramchandren R, Bartlett NL, Cheson BD, de Vos S, ForeroTorres A, Moskowitz CH, Connors JM, Engert A, Larsen EK, Kennedy DA, Sievers EL, Chen R (2012) Results of a pivotal phase II study of brentuximab vedotin for patients with relapsed or refractory Hodgkin's lymphoma. J Clin Oncol 30(18):2183-2189. doi:10. 1200/JCO.2011.38.0410 JCO.2011.38.0410

7. Rothe A, Sasse S, Goergen H, Eichenauer DA, Lohri A, Jager U, Bangard C, Boll B, von Bergwelt Baildon M, Theurich S, Borchmann P, Engert A (2012) Brentuximab vedotin for relapsed or refractory $\mathrm{CD} 30+$ hematologic malignancies: the German Hodgkin study group experience. Blood 120(7):1470-1472. doi:10. 1182/blood-2012-05-430918 blood-2012-05-430918

8. Gibb A, Jones C, Bloor A, Kulkarni S, Illidge T, Linton K, Radford J (2013) Brentuximab vedotin in refractory CD30+ lymphomas: a bridge to allogeneic transplantation in approximately one quarter of patients treated on a named patient programme at a single UK center. Haematologica 98(4):611-614. doi:10.3324/haematol.2012.069393 haematol.2012.069393

9. Zinzani PL, Viviani S, Anastasia A, Vitolo U, Luminari S, Zaja F, Corradini P, Spina M, Brusamolino E, Gianni AM, Santoro A, Botto B, Derenzini E, Pellegrini C, Argnani L (2013) Brentuximab vedotin in relapsed/refractory Hodgkin's lymphoma: the Italian experience and results of its use in daily clinical practice outside clinical trials. Haematologica 98(8):1232-1236. doi:10.3324/haematol.2012. 083048 haematol.2012.083048

10. Chen R, Palmer JM, Thomas SH, Tsai NC, Farol L, Nademanee A, Forman SJ, Gopal AK (2012) Brentuximab vedotin enables successful reduced-intensity allogeneic hematopoietic cell transplantation in patients with relapsed or refractory Hodgkin lymphoma. Blood 119(26):6379-6381. doi:10.1182/blood-2012-03-418673 blood2012-03-418673 